\section{ACKNOWLEDGMENTS}

This work was possible thanks to the Ligue Nationale Contre le Cancer (Comité de l'Isère) and the PHRC 1997. I.D. was supported by grants from the Ligue Nationale Contre le Cancer (Comité de la Drôme).

\section{REFERENCES}

1.Bernard, K., N. Auphan, S. Granjeau, G. Victorero, A.M. Schhmitt-Verhulst, B.R. Jordan, and C. Nguyen. 1996. Multiplex messenger assay: simultaneous, quantitative measurement of expression of many genes in the context of $\mathrm{T}$ cell activation. Nucleic Acids Res. 24:1435-1442.

2.Bertucci, F., K. Bernard, B. Loriod, Y.C. Chang, S. Grangeaud, D. Birnbaum, C. Nguyen, K. Peck, et al. 1999. Sensitivity issues in DNA array-based expression measurements and performance of nylon microarrays for small samples. Hum. Mol. Genet. 8:2129.

3.Bertucci, F., R. Houlgatte, A. Benziane, S. Grangeaud, J. Adelaïde, R. Tagett, B. Loriod, J. Jacquemier, et al. 2000. Gene expression profiling of primary breast carcinomas using arrays of candidate genes. Hum. Mol. Genet. 20:289-291.

4.Chambers, J., A. Angulo, D. Amaratunga, H. Guo, Y. Jiang, J. Wan, A. Bittner, K. Frueh, et al. 1999. DNA microarray of the complex human cytomegalovirus genome: profiling kinetic class with drug sensitivity of viral gene expression. J. Virol. 73:5757-5766.

5.El Atifi, M., I. Dupré, B. Rostaing, E.M. Chambaz, A.L. Benabid, and F. Berger. 2002. Long oligonucleotide arrays on nylon for large-scale gene expression analysis. BioTechniques 33:612-618

6.Jin, K., X. Mao, M.W. Eshoo, T. Nagayama, M. Minami, R.P. Simon, and D.A. Greenberg. 2001. Microarray analysis of hippocampal gene expression in global cerebral ischemia. Ann. Neurol. 50:93-103.

7.Kane, M.D., T.A. Jatkoe, C.R. Stumpf, J. Lu, J.D. Thomas, and S.J. Madore. 2000. Assessment of the sensitivity and specificity of oligonucleotide (50mer) microarrays. Nucleic Acids Res. 28:4552-4557.

Received 24 February 2003; accepted 9 May 2003.

Address correspondence to Michèle El Atifi, CHU Grenoble, Equipe Transcriptome, Pavillon B, BP 217, 38043 Grenoble Cedex 09, France.e-mail: melatifi@chu-grenoble.fr or to Isabelle Dupré, Laboratoire de neurosciences précliniques, INSERM U318, CHU Grenoble, Pavillon B, BP 217, 38043 Grenoble Cedex 09, France. e-mail: Isabelle.Dupre@ujf-grenoble.fr

\title{
Nonradioactive assay for quality control of flat-bottom pins during DNA-array production
}

\author{
Stefan Kotschote, Ulrike Teichmann, and Igor Ivanov \\ GPC Biotech AG, Munich, Germany
}

BioTechniques 35:266-270 (August 2003)

A number of recent publications have addressed issues related to the quality of array manufacturing reviewed by Holloway et al. (1) and especially the spotting tools used for the production of high-quality DNA arrays $(2,3)$. Variations in pin geometry cause variations in the transferred volume, which then affect the size of the spotted dots and ultimately the quality of the results. Individual analysis of the pins is very time-consuming, labor-intensive, and hardly applicable for the 384pin gadgets often used to produce DNA arrays. The quality of the pins used for spotting can be improved either by changing the material used to make the pins (2) or by preselecting the pins using a precise radioactive assay (3). Both publications pointed out that aging of the pins caused by multiple spotting may influence the pin geometry. Indirectly, the quality of the spots can provide an indication of pin quality, for example, by spiking fluorescent dyes to the spotting solution $(4,5)$, but the methods cannot be applied to, for example, nylon-based arrays. Here we suggest a method for the quality control of flat-bottom pins based on fluorescence measurement of the volumes transferred by individual pins. The procedure requires less than $30 \mathrm{~min}$ and can be applied for quality control of the pins if performed between or even during spotting runs.

As a first step, we established a method to measure reliably as little as 3 $\mu \mathrm{L}$ fluorescein solution in the $\mathrm{V}$-shaped 384-well microplate. The fluorescein solution was prepared by dissolving the amount of fluorescein powder (Molecular Probes, Eugene, OR, USA) required for a final concentration of $2.5 \mu \mathrm{g} / \mathrm{mL}$ in the dilution buffer containing $20 \mathrm{mM}$
Tris- $\mathrm{HCl}, \mathrm{pH}$ 8.0, 1.5× Q-solution (Qiagen, Hilden, Germany), and $10 \mathrm{mM}$ dithiothreitol as an anti-fading agent (6). The linearity of fluorescence detection was investigated by measuring different volumes of fluorescein solution in the V-shaped 384-well microplate (Genetix, New Milton, UK). Ninety-six wells of the plate filled with $3,6,9$, or $12 \mu \mathrm{L}$ fluorescein solution were measured using Wallac VICTOR ${ }^{2 \mathrm{TM}}$ multilabel counter (PerkinElmer Life Sciences, Zaventem, Belgium). The measured signals from each well were averaged over the 96 values obtained for each volume and normalized such that the signal measured at $12 \mu \mathrm{L}$ gave a value of 1 . As shown in Figure 1, the directly measured signals for 3 and $6 \mu \mathrm{L}$ are stronger than theory predicts. This is probably due to the V-shape format of the wells and/or uneven distribution of the liquid in the bottom of the wells. However, this effect can be reversed close to theoretical values by adding 20 $\mu \mathrm{L} 20 \mathrm{mM}$ Tris-HCl, pH 8.0, to every well ("fill up" line in Figure 1). We chose $20 \mu \mathrm{L}$ for simplicity of pipetting and the volume limits of the microplate.

The quality-control assay for the pins was designed to simulate the process that typically occurs during array production. The amount of the liquid in the microplate is sufficient to produce at least 160 arrays. The robot inoculates the pins, arrayed in a format complementary to the microplate, into the spotting solution and transfers the liquid onto the membrane. To counterbalance variations in transferred volumes and to increase sample (usually DNA) amount on the support, spotting is usually repeated at least four times at the same position on the array $(7,8)$. In addition, each array usually contains 
duplicate spots of the same sample. Therefore, during a production run, every pin has to contact the membrane surface and deposit liquid 1280 times (4 times deposition $\times 2$, for duplicates, $\times 160$ arrays).

To estimate the quality of the pins, we calculated the transferred volumes by measuring the changes in fluorescein amounts in wells of a microplate before and after spotting. With the exception of using fluorescein rather than DNA in the solution, we kept all parameters the same. We prepared two 384well microplates filled with $12 \mu \mathrm{L}$ fluorescein solution. One plate was used for deposition on the nylon membrane; the second was used as a control and kept in the robot environment without spotting. For spotting, we used flat-bottom pins $250 \mu \mathrm{m}$ in diameter (Genetix). After 1280 depositions, both plates were "filled up" with $20 \mu \mathrm{L}$ dilution buffer, and the fluorescence intensities were measured again.

The deposited volumes were calculated as:

$V_{i}=\frac{12 \mu L}{\overline{C_{\text {control }}}} *\left(C_{i}^{\text {before }}-C_{i}\right.$ after $\left.* N\right)$

where $C_{i}$ before and $C_{i}$ after are the fluorescence signals before and after spotting of the i-well and $N$ is a normalization factor between two measurements, which usually depends on the spotting time and adjustment of the detection instrument. $N$ was calculated as a ratio of the mean value of the control plate before spotting and after the "filling up" procedure. Typically, this value was $0.9-1.1$, adding about $10 \%$ systematic error to the calculations - in other words, a constant factor to the measured volumes of all pins. Moreover, the range of spotted volumes even with adjusted pins can vary about 2 -fold, as has been shown previously (3); therefore, the normalization factor could be neglected at the first approximation. We repeated the experiments three times and derived the average $\mathrm{V}_{\mathrm{i}}$ for every pin.

Similar to Soldatov et al. (3), we were able to rank pins according to their quality and select only the pins with very similar volume characteristics, as shown in Figure 2A. After

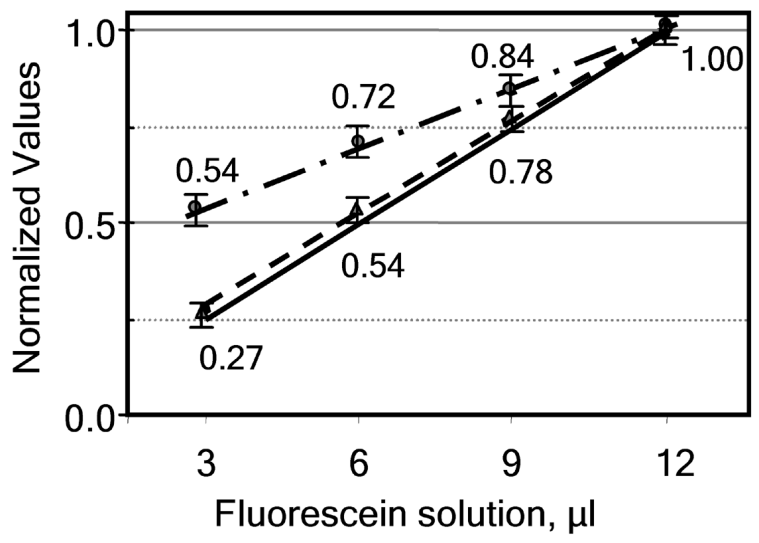

Figure 1. Measurement of fluorescein solution in V-shaped microplate. Fluorescein $(3,6,9$, and $12 \mu \mathrm{L})$ was deposited in 384-well microplates. Two sets of measurements were performed either directly or by adding $20 \mu \mathrm{L}$ Tris$\mathrm{HCl}$ ("fill-up"; see text). In total, 96 values for each sample volume were averaged, followed by normalization, setting the values for the $12-\mu \mathrm{L}$ starting volume at 1 . Theoretically, values for 9,6 , and $3 \mu \mathrm{L}$ should have been 0.75 , 0.5 , and 0.25 , respectively, as shown by the solid line. The actual results obtained for direct (dashed-dotted line) and fill up (dashed line) samples were plotted as best fitting lines to the measured values. The numbers at the lines indicate the normalized measured values, whereas the error bars correspond to the standard deviation of the measurements. testing 600 pins, we selected 432 pins of satisfactory quality and created two gadgets with 216 pins each. To measure the volumes transferred by each pin of the gadgets, we used the procedure described above, with the exception that the normalization factor was calculated using outer wells of the plate: the whole 384-well plate was filled with the fluorescein solution, but liquid from only the 216 central wells was spotted. As shown in Figure 2A, the average total volume of liquid transferred by the pin is $3.3 \pm 0.7 \mu \mathrm{L}$. Taking into account that this volume was transferred with 1280 depositions, one can calculate that the volume for individual transfer is
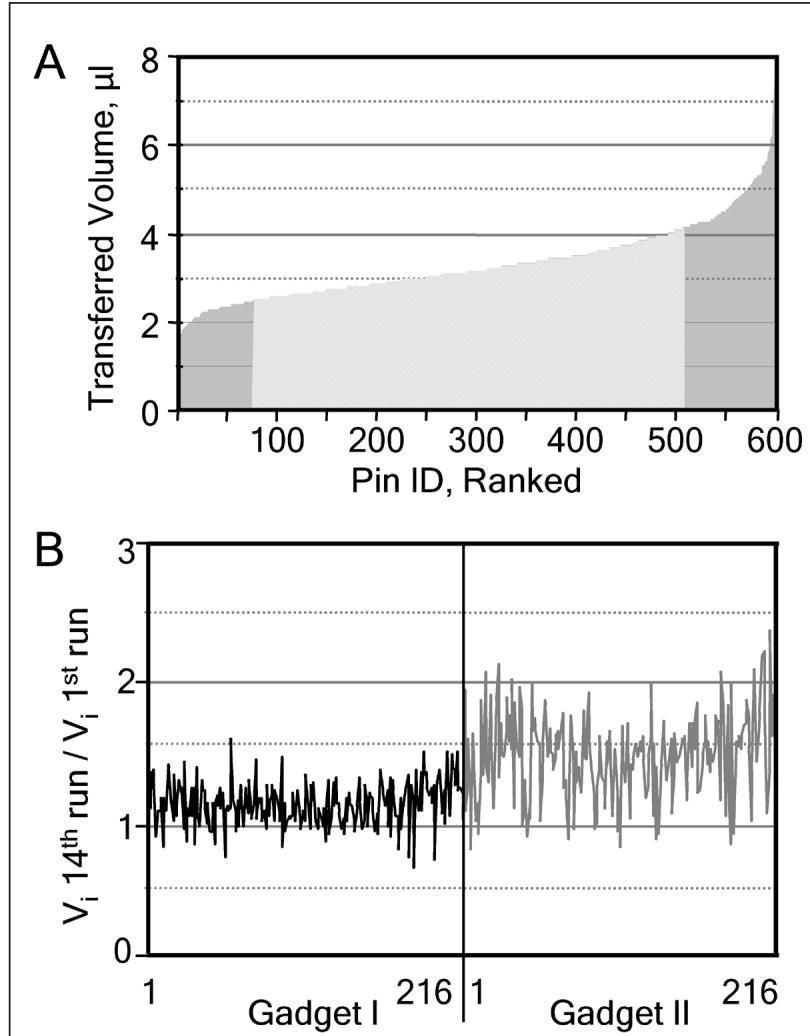

Figure 2. Estimation of the volumes transferred by pins as a qualitycontrol measure. (A) Transferred volumes of 600 flat-bottom pins were measured, and the values were ranked from lowest to highest value. Four hundred thirty-two pins, which showed the smallest difference to the average transferred volume of $3.3 \mu \mathrm{L}$ during a standard spotting run, were selected (hatched area) and used further for construction of two gadgets with a random selection of these pins. (B) Transferred volumes were compared after completing the first and fourteenth spotting runs. Ratios of fourteenth:first run values were calculated for every single pin on both gadgets. Ratios greater than 1 indicate that the pins transferred more volume after the fourteenth spotting run. On average, the transferred volume of all pins increased by a factor of 1.1 , with a CV equal to $14.8 \%$ (gadget I, left side) and a factor of $1.5 ; \mathrm{CV}=21.4 \%$ (gadget II, right side). 
about $2.6 \pm 0.6 \mathrm{~nL}$, which is very close to the $3.2 \mathrm{~nL}$ reported by Soldatov et al. (3). This value can be theoretically estimated as $4.1 \mathrm{~nL}$ from the assumption that the transferred volume is a semisphere with a diameter of $250 \mu \mathrm{m}$. Obviously, the lower volume measured indicates that the semi-sphere is not ideal but rather squashed in the center.

After the production of 2240 arrays (14 runs) with each gadget that corresponds to dotting 215,040 times with every pin, we reanalyzed the results of the volume transferred by individual pins. Figure $2 \mathrm{~B}$ shows the ratio of transferred volumes detected with "used" pins compared to "fresh" ones. In the ideal case, the ratio should be 1 , but the flat-bottom pins tend to deform during spotting runs, causing variations in pin diameter and thus transferred amount. As shown in Figure 2B, the ratio is 1.1 with a CV of $14.7 \%$ for the first gadget and 1.5 with a CV of $21.4 \%$ for the second one. Thus, although we started with the same pin population, aging of the pins obviously depends on pin assembly, robot settings, or other as yet unknown factors, stressing the fact that aging should be taken into account if large-scale spotting is planned.

One way to devise a tolerated limit for pin variations is to account for transferred volumes. For spotting, we use V-shaped wells filled with $12 \mu \mathrm{L}$ DNA solution. To avoid damaging the pins in the microplate, we can effectively use only $10 \mu \mathrm{L}$ of the volume for spotting. Figure $2 \mathrm{~A}$ shows that the highest total transferred volume of the selected pins spotted from one plate is about $4 \mu \mathrm{L}$ and the lowest volume is about $2.5 \mu \mathrm{L}$, a similar range to that reported earlier (3). To be sure that we evenly deposit DNA on the array, we only use pins that cannot transfer more than $9 \mu \mathrm{L}$ solution (or a factor of 2.3 in Figure 2B). If the value for one of the pins exceeds this parameter, then we substitute this pin by a previously tested one. It is worth mentioning that the major effect of pin variation manifests in different spot size estimations by image analysis software and as a consequence in evaluation of the neighborhood effect $(9,10)$. The latter procedure relies on even and precise positioning of different DNA fragment samples at high density on a solid support. The variations stemming from the same pin on different arrays are usually accounted for by applying correction procedures in combination with different methods of normalization (11).

The method described here can easily be implemented by any laboratory involved in production of nylon-, plastic- (12), or glass-based (8) arrays using flat-bottom pins. It can help in monitoring the consistency of the process (e.g., pin aging) and in adjusting the liquid volume needed for spotting, which can contribute to cost savings. The major advantages of the method are its speed and nonradioactive character, meaning it can be carried out between different robotic runs. We recently found that the method can be further simplified if the DNA used for spotting is directly "spiked" with fluorescent dye followed by the measurements described above. This method is different from those described earlier $(4,5)$, although it only provides estimates of the DNA amount deposited on the membrane rather than direct quality control of spots used by the methods citied earlier.

\section{ACKNOWLEDGMENTS}

We thank Matthias Borgmann for help in setting up fluorescence assays, Oliver Herde and Franz Steinmaßl for assistance in robot handling, Frank Becker for useful suggestions, and Avril Arthur-Goettig for adding valuable suggestions and corrections to the text. The work was supported by Eureka grant $\Sigma ! 2370$ from the Federal Ministry of Research and Education (BMBF, Germany). The current address for U.T. is Micromet AG, Staffelseestr. 2, 81447, Munich, Germany.

\section{REFERENCES}

1.Holloway, A.J., R.K. van Laar, R.W. Tothill, and D.D. Bowtell. 2002. Options availablefrom start to finish - for obtaining data from DNA microarrays II. Nat. Genet. 32(Sup$p l): 481-489$.

2.George, R.A., J.P. Woolley, and P.T. Spellman. 2001. Ceramic capillaries for use in microarray fabrication. Genome Res. 11:1780 1783.

3.Soldatov, A.V., E.N. Nabirochkina, S.G. Georgieva, and H. Eickhoff. 2001. Adjustment of transfer tools for the production of micro- and macroarrays. BioTechniques 31 : 848-854.

4.Diehl, F., B. Beckmann, N. Kellner, N.C. Hauser, S. Diehl, and J.D. Hoheisel. 2002. Manufacturing DNA microarrays from unpurified PCR products. Nucleic Acids Res. 30:e79.

5.Shearstone, J.R., N.E. Allaire, M.E. Getman, and S. Perrin. 2002. Nondestructive quality control for microarray production. BioTechniques 32:1051-1057.

6.Picciolo, G.L. and D.S. Kaplan. 1984. Reduction of fading of fluorescent reaction product for microphotometric quantitation. Adv. Appl. Microbiol. 30:197-234.

7.Eickhoff, H., I. Ivanov, M. Kietzmann, E. Maier, M. Kalkum, D. Bancroft, and $\mathbf{H}$. Lehrach. 1999. Robotic equipment and microsystem technology in biological research, p. 17-30. In J.M. Köhler, T. Mejevaia, and H.P. Saluz (Eds.), Microsystem Technology: A Powerful Tool for Biomolecular Studies. Birkhäuser Verlag, Berlin.

8.Eickhoff, H., J. Schuchhardt, I. Ivanov, S. Meier-Ewert, J. O'Brien, A. Malik, N. Tandon, E.W. Wolski, et al. 2000. Tissue gene expression analysis using arrayed normalized cDNA libraries. Genome Res. 10:1230-1240.

9.Therneau, T., R.C. Tschumper, and D. Jelinek. 2002. Sharpening spots: correcting for bleedover in cDNA array images. Math. Biosci. 176:1-15.

10.Machl, A.W., C. Schaab, and I. Ivanov. 2002. Improving DNA array data quality by minimising 'neighbourhood' effects. Nucleic Acids Res. 30:e127.

11.Schuchhardt, J., D. Beule, A. Malik, E. Wolski, H. Eickhoff, H. Lehrach, and H. Herzel. 2000. Normalization strategies for cDNA microarrays. Nucleic Acids Res. 28:E47.

12.Munishkin, A., K. Faulstich, V. Aivazachvili, C. Granger, and A. Chenchik. 2003. Plastic microarrays: a novel array support combining the benefits of macro- and microarrays. Methods Mol. Biol. 224:31-54.

Received 10 April 2003; accepted 14 May 2003.

Address correspondence to Igor Ivanov, GPC Biotech AG, Fraunhofer str. 20, 82152, Martinsried/Munich, Germany. e-mail:igorivanov@gpc-biotech.com 